\title{
In Situ Studies of Phase Transitions in Thin Discotic Films
}

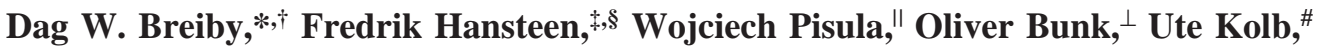 \\ Jens W. Andreasen, ${ }^{\dagger}$ Klaus Miillen," and Martin M. Nielsen ${ }^{\dagger}$ \\ Danish Polymer Centre, Risф National Laboratory, P.O. Box 49, 4000 Roskilde, Denmark, IMM, \\ Radboud University Nijmegen, Toernooiveld 1, 6525 ED Nijmegen, The Netherlands, \\ The Norwegian University of Science and Technology, 7491 Trondheim, Norway, \\ Max-Planck-Institute for Polymer Research, Ackermannweg 10, 55128 Mainz, Germany, Paul Scherrer Institut, \\ 5232 Villigen PSI, Switzerland, and Institut für Physikalische Chemie, Johannes Gutenberg-Universität Mainz, \\ Welderweg 11, 55099 Mainz, Germany
}

Received: September 1, 2005

\begin{abstract}
The crystalline to liquid crystalline $(\mathrm{Cr}-\mathrm{LC})$ phase transition in thin films of zone-cast hexa-perihexabenzocoronene sixfold substituted with dodecyl side chains $\left(\mathrm{HBC}-\mathrm{C}_{12} \mathrm{H}_{25}\right)$ has been studied in detail using grazing incidence X-ray diffraction (GID), electron diffraction (ED), and variable angle spectroscopic ellipsometry (VASE), When heating the material, a first minor transition is observed around $42{ }^{\circ} \mathrm{C}$. This change is attributed to alterations of the crystalline alkyl chain packing, which only slightly changes the electronic properties of the material. At higher temperatures of about $90{ }^{\circ} \mathrm{C}$, but still significantly below the previously reported transition temperature in bulk, the $\mathrm{Cr}-\mathrm{LC}$ transition is observed. An accompanying large increase in optical anisotropy is compatible with the X-ray data, showing a transition from the as-cast herringbone-like crystalline state to a highly ordered discotic hexagonal columnar LC phase. The structural transition has the macroscopic effect of increasing the film thickness. The high structural order of the as-cast low-temperature phase is only partly recovered after cooling, and the phase transition exhibits a large hysteresis. From the ellipsometry data, the dielectric tensor of $\mathrm{HBC}-\mathrm{C}_{12} \mathrm{H}_{25}$ was refined to unprecedented detail.
\end{abstract}

\section{Introduction}

Organic electronics have promising prospects, with the main motivation being to make cheap plastic sensors and circuits. ${ }^{1}$ The importance of well-defined molecular order to exploit the highly anisotropic properties of the specifically designed molecules is well-recognized. A requirement for good mobility in films of organic conjugated molecules is a good overlap of the $\pi$-electrons of neighboring molecules. Many pathways are being explored to achieve self-assembled highly connected networks of $\pi-\pi$ interacting molecules, including both chemical modifications $^{2-4}$ and processing techniques..$^{5-12}$

Discotics, i.e., planar, disc-shaped aromatic molecules, have been intensely studied during the past two decades. To make them soluble and to control the thermal properties, they are substituted with side chains at the periphery. The molecules tend to segregate into stacked columns of the aromatic cores, separated by less-ordered side chains. These stacks can be considered one-dimensional molecular wires, whereas the side chains, typically alkyl, are electronically inactive. A relatively high mobility of typically $1.0 \mathrm{~cm}^{2} / \mathrm{V}$ s is obtained in these materials. ${ }^{13}$ The molecules studied here, hexa-peri-hexabenzocoronenes (HBC; see Figure 1) are graphenes, having delocalized electrons on both sides of the molecular plane. Underivated $\mathrm{HBC}$ is highly crystalline and poorly soluble. Because of electrostatic quadrupolar interactions, the molecules tend to pack in the so-called herringbone structure.

\footnotetext{
† Danish Polymer Centre.

$\doteqdot$ Radboud University Nijmegen.

$\S$ The Norwegian University of Science and Technology.

" Max-Planck-Institute for Polymer Research.

$\perp$ Paul Scherrer Institut.

\# Institut für Physikalische Chemie.
}

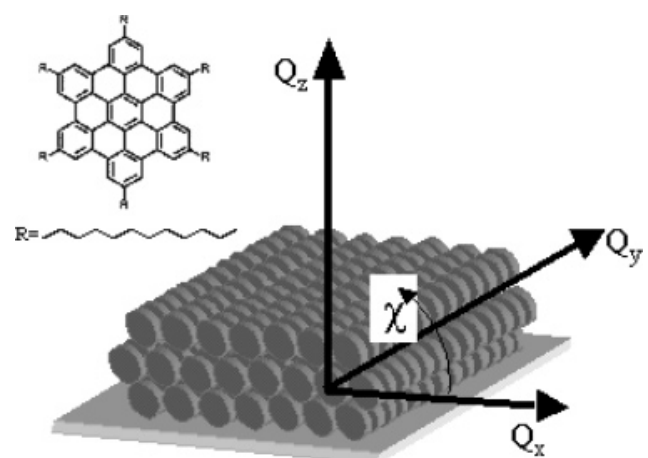

Figure 1. Sketch of discotics arranged in columns parallel to the substrate and the definition of the reciprocal coordinate system $\left(Q_{x}\right.$, $Q_{y}, Q_{z}$ ). The angle $\chi$ defines a rotation about $Q_{y}$. The chemical structure of $\mathrm{HBC}-\mathrm{C}_{12} \mathrm{H}_{25}$ is also shown.

Few discotics are liquid crystalline at room temperature. ${ }^{14}$ However, upon heating, the structure of many discotics changes from a crystalline to a columnar liquid-crystalline mesophase, with the transition temperature often around $100-200{ }^{\circ} \mathrm{C}$. Differential scanning calorimetry (DSC) studies of bulk HBC$\mathrm{C}_{12} \mathrm{H}_{25}$ samples ${ }^{15}$ have shown a considerable change in the columnar structure from the crystalline phase to the mesophase at a phase transition temperature of $107{ }^{\circ} \mathrm{C}$. The discotic molecules changed the intracolumnar packing from a crystalline herringbone structure to a hexagonal columnar liquid-crystalline phase with cofacially arranged stacked cores.

A detailed structural analysis of ultrathin zone-cast HBC$\mathrm{C}_{12} \mathrm{H}_{25}$ films in the crystalline phase has recently been carried out using synchrotron grazing incidence diffraction. ${ }^{16}$ The unit cell was found to be orthorhombic, containing two molecules 
arranged in a herringbone pattern with the discotics orienting edge-on with respect to the substrate. The dodecyl side chains were found to be in a highly ordered state, leading to the formation of a vertical lamellar superstructure with aromatic and aliphatic fractions. ${ }^{16}$ In earlier articles on the optical properties of ordered $\mathrm{HBC}-\mathrm{C}_{12} \mathrm{H}_{25}$ films above and below $T_{\mathrm{LC}}$, it has been suggested that a much higher optical anisotropy in the LC phase could be due to a change of tilt from a herringbone structure to a cofacial arrangement. ${ }^{17,18}$ This thermal behavior will be addressed in detail in this article.

To study the crystalline to liquid crystalline $(\mathrm{Cr}-\mathrm{LC})$ phase transition in zone-cast $\mathrm{HBC}-\mathrm{C}_{12} \mathrm{H}_{25}$ films, separate experiments were carried out using variable angle spectroscopic ellipsometry (VASE), electron diffraction (ED), and grazing incidence X-ray diffraction (GID), thus allowing monitoring of both the optical (electronic) and structural temperature dependence. $\mathrm{A} \mathrm{Cr}-\mathrm{Cr}$ transition was found at about $42^{\circ} \mathrm{C}$. The $\mathrm{Cr}-\mathrm{LC}$ phase transition of the zone-cast films is shifted significantly toward a lower temperature compared with bulk samples and exhibits a large degree of hysteresis.

\section{Experimental Section}

Sample Preparation. The $\mathrm{HBC}-\mathrm{C}_{12} \mathrm{H}_{25}$ was synthesized as described elsewhere. ${ }^{19}$ For obtaining the films, zone casting was employed, ${ }^{20}$ giving a high degree of in-plane alignment without using aligning substrates, stretching, or rubbing. The resulting films are thin, transparent, homogeneous, and with a highly reproducible biaxially oriented crystalline structure. ${ }^{20,16}$

Grazing Incidence X-ray Diffraction (GID). The GID studies were performed using the $z$-axis diffractometer at the wiggler beamline BW2 at Hasylab. The wavelength $\lambda$ was $1.2398 \AA$, corresponding to a photon energy of $10 \mathrm{keV}$. GID studies are performed with the beam impinging onto the sample surface at a grazing incidence angle (here, $0.16^{\circ}$ ), giving total reflection from the glass substrate. As a result, the (background) scattering from the substrate is strongly reduced, effectively enhancing the signal from the thin organic film. ${ }^{21}$ A Cyberstar point detector was used to collect the scattered intensity. The sample cell was made of X-ray transparent Kapton and flushed with helium to reduce background and radiation damage of the sample. The sample was mounted on a temperature-controlled $\left( \pm 1^{\circ} \mathrm{C}\right)$ copper block using glue and heat paste.

The scattering vector is defined by $\mathbf{Q} \equiv \mathbf{k}_{\text {out }}-\mathbf{k}_{\text {in }}$, with units of reciprocal angstroms. $\mathbf{k}_{\text {in }}$ and $\mathbf{k}_{\text {out }}$ denote the in- and outgoing wave vectors, respectively. For elastic scattering, $\left|\mathbf{k}_{\text {in }}\right|=\left|\mathbf{k}_{\text {out }}\right|$ $=2 \pi / \lambda$. The real space lattice spacing $d$ is related to $\mathbf{Q}$ by $d=$ $2 \pi / Q$, and we define $Q^{2}=Q_{x}{ }^{2}+Q_{y}{ }^{2}+Q_{z}{ }^{2} \cdot Q_{z}$ is oriented along the film normal. $Q_{x}$ and $Q_{y}$ are both in-plane, being perpendicular to and parallel to the molecular stacks, respectively, cf., Figure 1. We define $\chi$ to be the angle between $\mathbf{Q}$ and the $Q_{x}-Q_{y}$ plane. A $\chi$-scan starts at $Q_{x}=Q$ and moves on a circle segment toward $Q_{z}=Q$, thus measuring the degree of orientation about the $Q_{y}$ axis. $\chi=90^{\circ}$ cannot be reached by the GID technique. In addition to the $\left(Q_{x}, Q_{y}, Q_{z}\right)$ values giving absolute coordinates in reciprocal space, we employ Miller indices $h k l$.

Ellipsometry. Optical measurements were carried out using a variable angle spectroscopic ellipsometer (V-VASE) from J. A. Woollam Co., based on the rotating analyzer principle. ${ }^{20}$ A temperature-controlled heat cell purged with inert He gas was used for all measurements. The sample was oriented with the cast direction of the film at $45^{\circ}$ with respect to the plane of incidence inside the heat cell. Measurements were done for photon energies of $1-5 \mathrm{eV}$ at an incidence angle of $70^{\circ}$, close to the pseudo-Brewster angle of the sample. ${ }^{22}$
Ellipsometry is a highly sensitive optical measurement technique based on analyzing the change in polarization of a beam of light after reflection from or transmission through a sample. In comparison with UV-vis absorption spectroscopy, much more detailed information can be obtained by this technique. This advantage stems from simultaneous measurement of both amplitude ratio and relative phase of $\mathrm{p}$ - and s-polarized light (two parameters), in contrast to measuring only the intensity attenuation (one parameter) in the case of absorption spectroscopies.

In the Jones matrix formalism, ${ }^{22}$ the electric field of the reflected light $\mathbf{E}^{\prime}$ is related to the incident field $\mathbf{E}$ by a reflection matrix which characterizes the optical response of the sample

$$
\left(\begin{array}{l}
E_{\mathrm{p}}^{\prime} \\
E_{\mathrm{s}}^{\prime}
\end{array}\right)=\left(\begin{array}{ll}
r_{\mathrm{pp}} & r_{\mathrm{ps}} \\
r_{\mathrm{sp}} & r_{\mathrm{ss}}
\end{array}\right)\left(\begin{array}{l}
E_{\mathrm{p}} \\
E_{\mathrm{s}}
\end{array}\right)
$$

The subscripts $\mathrm{p}$ and $\mathrm{s}$ denote the components of the field polarized parallel to and perpendicular to the plane of incidence, respectively. In the special case of isotropic samples, the offdiagonal elements $r_{\mathrm{ps}}$ and $r_{\mathrm{sp}}$ are zero. The result of an ellipsometric measurement then yields the quantities $\Psi$ and $\Delta$, which are related to the amplitude reflection coefficients $r_{\mathrm{pp}}$ and $r_{\mathrm{ss}}$ for $\mathrm{p}$ - and s-polarized light by the fundamental equation of ellipsometry

$$
\frac{r_{\mathrm{pp}}}{r_{\mathrm{ss}}}=\tan (\Psi) \mathrm{e}^{i \Delta}
$$

In the case of reflection from bulk materials, $r_{\mathrm{pp}}$ and $r_{\mathrm{ss}}$ are the single interface Fresnel factors, and the optical constants of the medium can easily be calculated from the measured $\Psi$ and $\Delta$.

For more complex samples such as thin films and multilayer structures, the reflection coefficients are complicated functions of the thickness and the optical properties of the individual layers. With sufficient prior knowledge of the sample under study, a reliable mathematical model can be constructed and unknown parameters determined by numerical fitting to the ellipsometric measurement data, a procedure known as data inversion. Performing measurements at several angles of incidence as well as in a broad spectral region often benefits the inversion, as more information is made available for the numerical fitting. However, for bulk samples or in the present case of a very thin film on a substrate, multiple angles do not significantly increase the available information. Sample properties that are commonly deduced from ellipsometry measurements include layer thicknesses, surface and interfacial roughness and optical constants of thin films or bulk materials.

For anisotropic samples, additional quantities $\Psi_{\mathrm{ps}}$ and $\Delta_{\mathrm{ps}}$ can be defined. They represent the relative amplitude and phase of s-polarized light transformed into p-polarized light by the sample and may serve as a measure of optical anisotropy. By analogy to eq 2 , we define

$$
\frac{r_{\mathrm{ps}}}{r_{\mathrm{pp}}}=\tan \left(\Psi_{\mathrm{ps}}\right) \mathrm{e}^{i \Delta_{\mathrm{ps}}}
$$

The ellipsometer can measure $\Psi_{\mathrm{ps}}$ and $\Delta_{\mathrm{ps}}$ by appropriately setting the polarization of the incident light. By including anisotropic layers in the mathematical model, one can then determine the optical constants of anisotropic materials.

Electron Diffraction. Electron Diffraction (ED) was performed using a Philips EM-420 transmission electron microscope equipped with a LaB6 cathode, operating at an acceleration voltage of $120 \mathrm{kV}$. For the ED experiments, the film was 

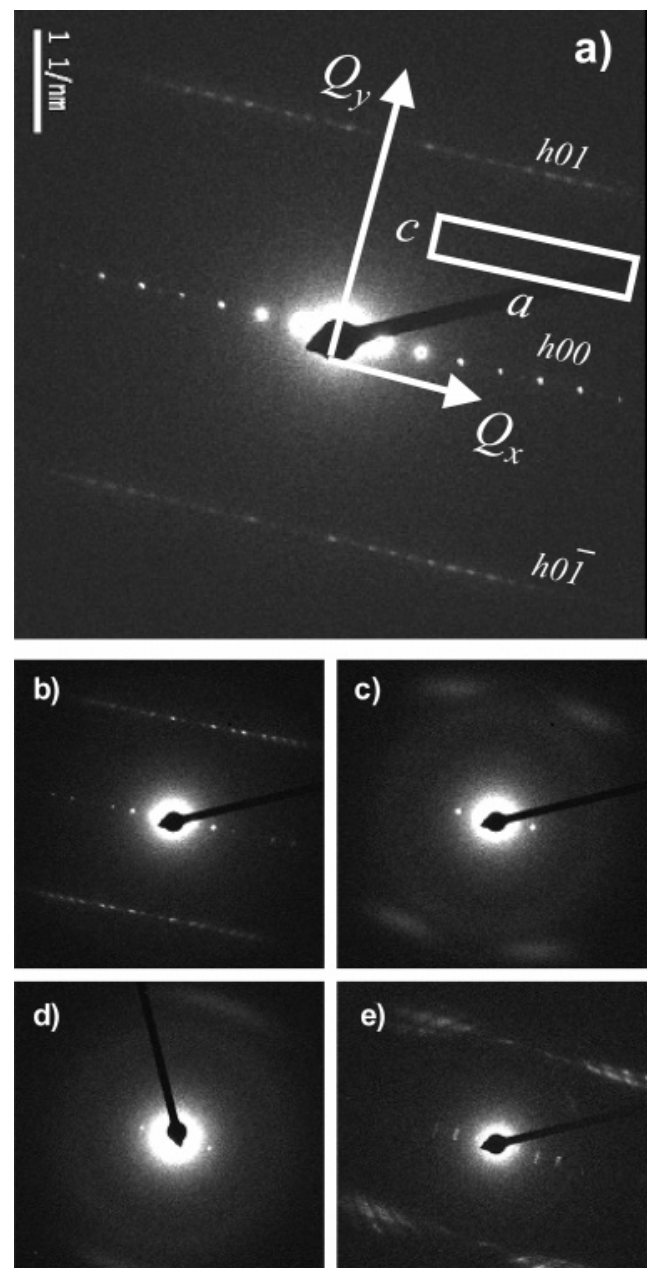

Figure 2. Temperature-dependent electron diffraction of zone-cast $\mathrm{HBC}-\mathrm{C}_{12} \mathrm{H}_{25}$ layers: image (a) taken at room temperature (crystalline phase), (b) at $40{ }^{\circ} \mathrm{C}$, (c) just above the phase transition, (d) at $130{ }^{\circ} \mathrm{C}$ (mesophase), and (e) after cooling back to room temperature. The arrows in (a) indicate the $Q_{x}$ and $Q_{y}$ directions, and the rectangle illustrates the orientation of the orthorhombic unit cell.

cast onto a carbon film evaporated onto a glass substrate. Subsequently, the $\mathrm{HBC}-\mathrm{C}_{12} \mathrm{H}_{25}$ /carbon film was removed from the glass using a dilute aqueous $\mathrm{HF}$ solution and mounted on a transmission electron microscopy (TEM) copper grid.

\section{Results}

Diffraction Results. The electron diffraction images in Figure $2 \mathrm{a}$, taken at room temperature, is fully consistent with previous reports. ${ }^{12,16}$ It provides information about the in-plane inter- and intracolumnar periodicities, indicating high crystallinity and pronounced long-range order of the zone-cast $\mathrm{HBC}-\mathrm{C}_{12} \mathrm{H}_{25}$. The intensity distribution of the diffraction peaks is consistent with an orthorhombic lattice with $a=49.4 \AA$ and $c=4.96 \AA$. The $b$-axis is out-of-plane, with $b=19.6 \AA$. The intensity distribution has been simulated and found to be consistent with a herringbone organization of the discotic molecules with a tilting angle of about $40^{\circ},{ }^{16}$ comparable to the $45^{\circ}$ reported for bulk HBC. ${ }^{12}$ The molecules stack in the $c$ direction, and the $a-c$ plane is parallel to the substrate. A remarkable feature of the low-temperature data is that they show a tremendous degree of order, which is interpreted as crystalline packing of both the aromatic HBC cores and the aliphatic side chains. ${ }^{16}$

To obtain a deeper insight into the change in the supramolecular organization during heating of the zone-cast layers to the mesophase, stepwise temperature increments were monitored using electron diffraction. At $T \approx 40{ }^{\circ} \mathrm{C}$, the gross features of the pattern observed at room temperature are preserved, cf., Figure 2b. Most importantly, the $h 01$ line of mixed index reflections is still present, indicating that long-range order is preserved. However, the reflection intensities have changed significantly, suggesting a different, yet related, structure. This is thus a $\mathrm{Cr}-\mathrm{Cr}$ transition.

At the $\mathrm{Cr}-\mathrm{LC}$ phase transition at higher $T$, the structure of the zone-cast film changes abruptly and completely to a tilted columnar LC structure. The characteristic diffraction lines of the crystalline structure disappear, and a pattern compatible with short-range liquid crystalline order can be seen in Figure 2c. The changes are most pronounced for the mixed index reflections, with the $h 01$ line transformed into two weak offmeridional reflections. With further heating, these two offmeridional peaks merge, resulting finally well above the phase transition in a single meridional peak corresponding to a $\pi-\pi$ stacking distance of $3.5 \AA$, cf., Figure 2 d. In the mesophase, only a few equatorial reflections corresponding to $25.2 \AA$ can be observed, as further discussed below. The diffuse rings observed in the LC phase might correspond to disordered alkyl chains.

After cooling back to room temperature, the high initial order of the surface layer is not completely recovered. The diffraction pattern suggests a columnar structure of tilted molecules, probably the herringbone arrangement of the as-cast phase. However, the reflection peaks are significantly broader and smeared, suggesting a higher disorder of the molecules. Whereas the as-cast sample showed a highly homogeneous structure, the disorder is increased after visiting the mesophase. Parts of the films still possess a well-ordered alignment, but others exhibit considerable columnar misalignment, cf., Figure 2e.

Whereas ED in a single exposure rapidly gives an overview of the in-plane reflections as in Figure 2, GID has the advantages of yielding more quantitative results and not being limited to in-plane diffraction. Repeated in-plane GID scans along $Q_{x}$ for $Q_{y}=0 \AA^{-1}(k=0)$ and $Q_{z}=0.03 \AA^{-1}(1 \sim 0)$ are shown in Figure 3 . These scans were performed to complement and extend the ED data in Figure 2. Between each scan, the temperature was increased stepwise with increments of a few degrees. The low-temperature scans are consistent with previous diffraction results. ${ }^{16}$ A first structural change is observed at $\sim 43{ }^{\circ} \mathrm{C}$, characterized by the almost complete disappearance of the weak reflections $h 00$ for $h$ odd.

At a high temperature of about $90{ }^{\circ} \mathrm{C}$, the as-cast structure is completely erased, however with the important exception that the overall alignment of the molecules persists, cf., Figures 2 and 3. When no further change of the GID diffractograms was observed upon increasing the temperature, the temperature was kept steady at $\sim 95^{\circ} \mathrm{C}$ and further data collected. The mixed index reflections with $l \neq 0$ are all absent. This implies that the registry between molecules in different columns has vanished, as expected for the liquid crystalline state. Note that, as for the ED results in Figure 2, there is a large hysteresis, and the initial structure is not fully recovered upon cooling back to room temperature.

GID measurements of the intercolumnar reflections at $\sim 95$ ${ }^{\circ} \mathrm{C}$ in the LC phase are shown in Figure 4, with findings being fully consistent with a hexagonal columnar LC phase. These "rocking scans" (constant $Q$ ) of the few remaining peaks in the $Q_{x}-Q_{z}$ plane (i.e., perpendicular to the columns) have maxima at $\chi=0^{\circ}, 30^{\circ}, 60^{\circ}$, and $90^{\circ}$ and exhibit a surprisingly narrow distribution, $\mathrm{fwhm}_{\chi} \approx 7^{\circ}$, cf., Figure 4 . The 100 reflection was found to have its maximum intensity for the scattering vector 


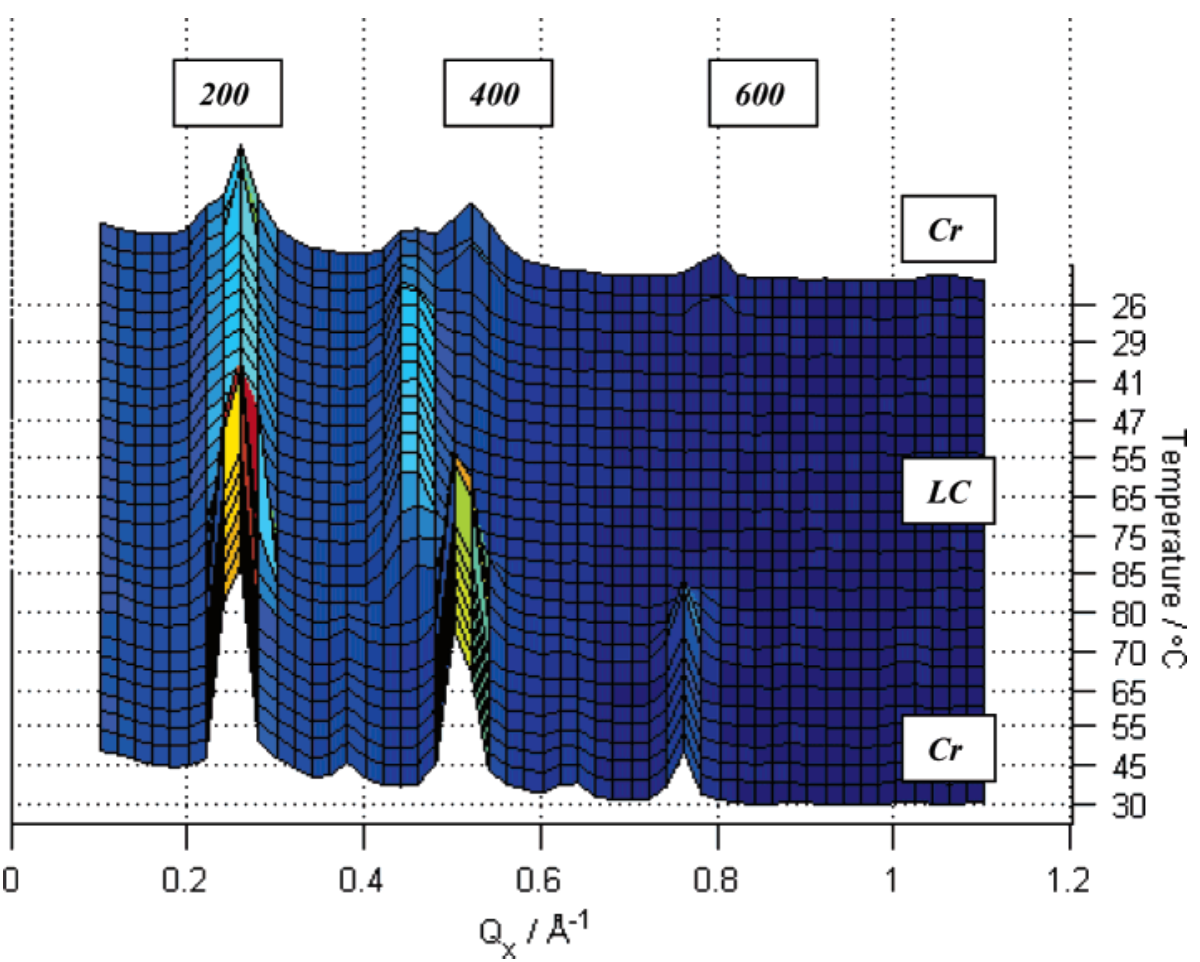

Figure 3. In situ annealing observed with GID. Repeated scans along the $Q_{x}$ axis $\left(Q_{y}=Q_{z} \approx 0\right)$ for different temperatures are shown, starting at the bottom of the plot. The indices are for the as-cast phase.

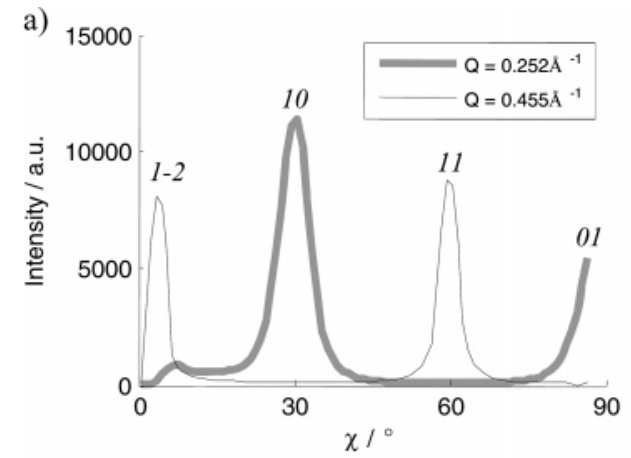

b)

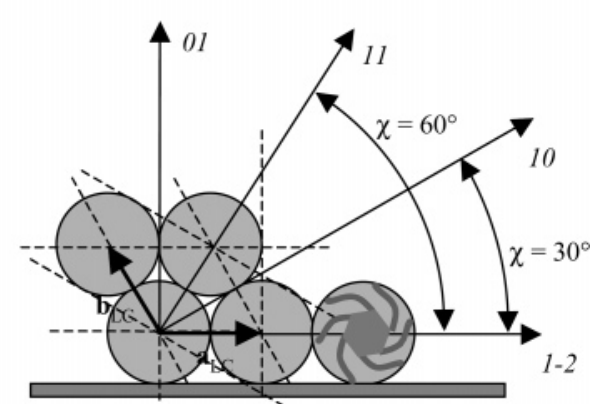

Figure 4. (a) GID rocking scans (constant $Q$ ) probing the crystallite orientation in the LC (meso-) phase. The peaks are at exactly $0^{\circ}, 30^{\circ}$, $60^{\circ}$, and $90^{\circ}$, being evidence for a hexagonal columnar phase. The line with the $\{110\}$ reflections is multiplied by 10 ; these peaks are almost extinct. (b) Interpretation of the diffraction results, with $\mathbf{a}_{\mathrm{LC}}$ and $\mathbf{b}_{\mathrm{LC}}$ being unit-cell vectors in the LC phase. The narrow widths of the peaks in (a) indicate a narrow orientation distribution of the ordered regions about the columnar axes, with the columns having a densely packed plane toward the substrate. For one of the cylindrical columns, the aromatic core and the side chains are schematically indicated.

forming a $\chi=30^{\circ}$ angle with the substrate. The $Q$ value of this peak is $0.252 \AA^{-1}$, corresponding to a real space lattice spacing of $24.9 \AA$ and an intercolumnar distance $a_{\mathrm{LC}}=27.8 \AA$. The columns are closely packed toward the substrate, as illustrated in Figure 4b. As is clear from Figure 4, there is also a 110 peak at $60^{\circ}$, however with a much lower intensity. That the 110 peak is almost extinct is expected for this centrosymmetric molecular arrangement.

Ellipsometry Results. In Figure 5, temperature and spectral dependence of the optical anisotropy represented by $\Psi_{\mathrm{ps}}$ is plotted. The structural phase transition taking place at $T \approx 90$ ${ }^{\circ} \mathrm{C}$ increases the optical anisotropy across the whole spectrum except at $3.8 \mathrm{eV}$. The signal is particularly strong at high photon energies, but also exhibits a peak at $3.4 \mathrm{eV}$. Note that the anisotropy keeps increasing with higher $T$ well above the phase transition.

The model used to invert the data consists of a silicon substrate with a thin oxide layer and a uniaxial anisotropic layer with its optical axis oriented in the plane of the film and parallel to the casting direction. Surface roughness effects had to be excluded from the model because of strong parameter correlations. By fitting the experimental data to this model, we obtained the film thickness as well as the qualitative spectral behavior of the complex refractive indices $N_{\|}$and $N_{\perp}$, parallel and perpendicular to the casting direction.

The film thickness was determined by considering the lowenergy region of the spectrum $1.5-2.5 \mathrm{eV}$ where the optical absorption of HBC is very low. A single parameter dispersion model (constant value) was used for the refractive indices in this spectral range. Attempting to introduce more parameters in the form of a Cauchy dispersion model failed because of strong parameter correlations in the weakly dispersive film. Best fits of the film thickness $d_{\mathrm{f}}^{\mathrm{Cr}}=19.5 \mathrm{~nm}$ for the $T=25^{\circ} \mathrm{C}$ low-temperature phase and $d_{\mathrm{f}}^{\mathrm{LC}}=23.5 \mathrm{~nm}$ in the $T=120^{\circ} \mathrm{C}$ high-temperature LC phase were found. These values are in good agreement with values obtained by atomic force microscopy (AFM). ${ }^{20}$ The origin of this increase of thickness is discussed later.

Keeping the film thickness fixed, $N_{\|}=n_{\|}+\mathrm{i} k_{\|}$and $N_{\perp}=$ $n_{\perp}+\mathrm{i} k_{\perp}$ were fitted point by point across the measured spectral region; see Figure 6 . The accuracy of the obtained optical 


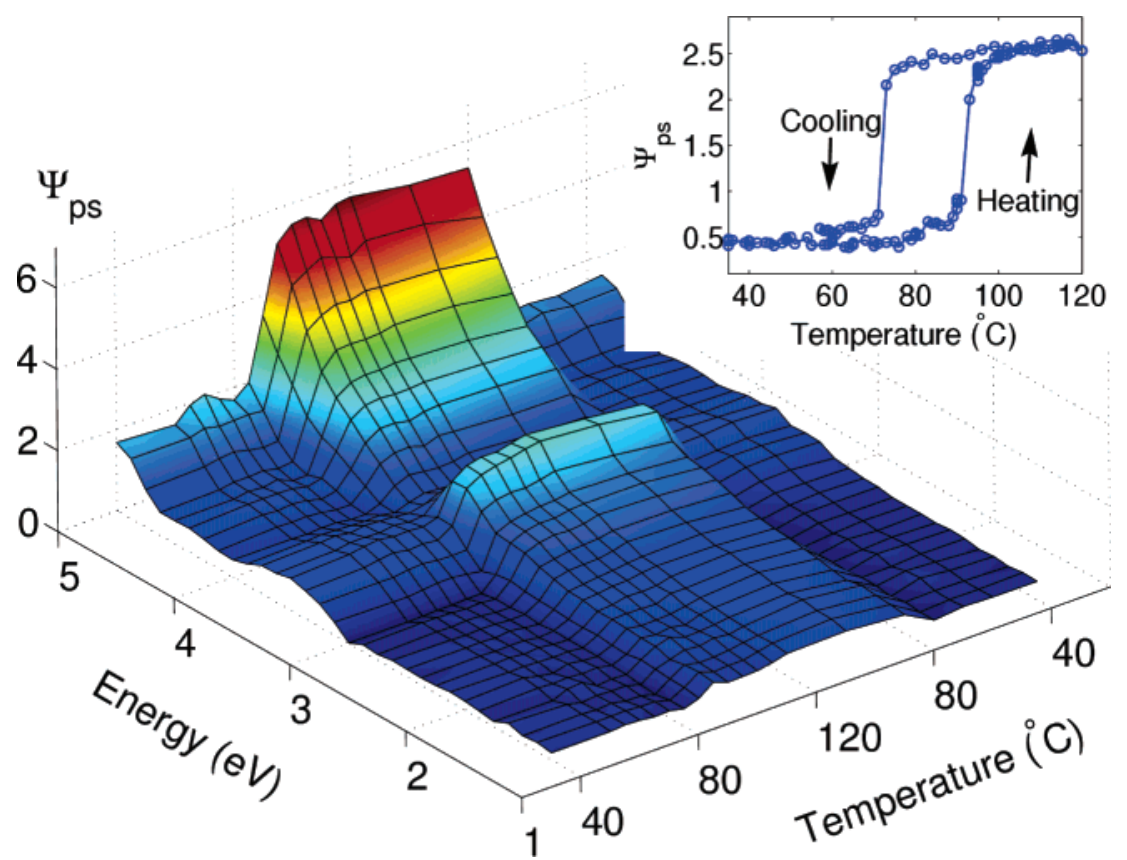

Figure 5. Optical anisotropy $\psi_{\mathrm{ps}}$ as a function of photon energy and temperature. The effect of the phase transition on the optical anisotropy can be seen across almost the whole measured spectrum, with the exception of a region at about $3.8 \mathrm{eV}$. The strongest influence on anisotropy is observed at $3.4 \mathrm{eV}$ and above $3.8 \mathrm{eV}$. The inset shows the temperature hysteresis of the phase transition measured at $3.4 \mathrm{eV}$.
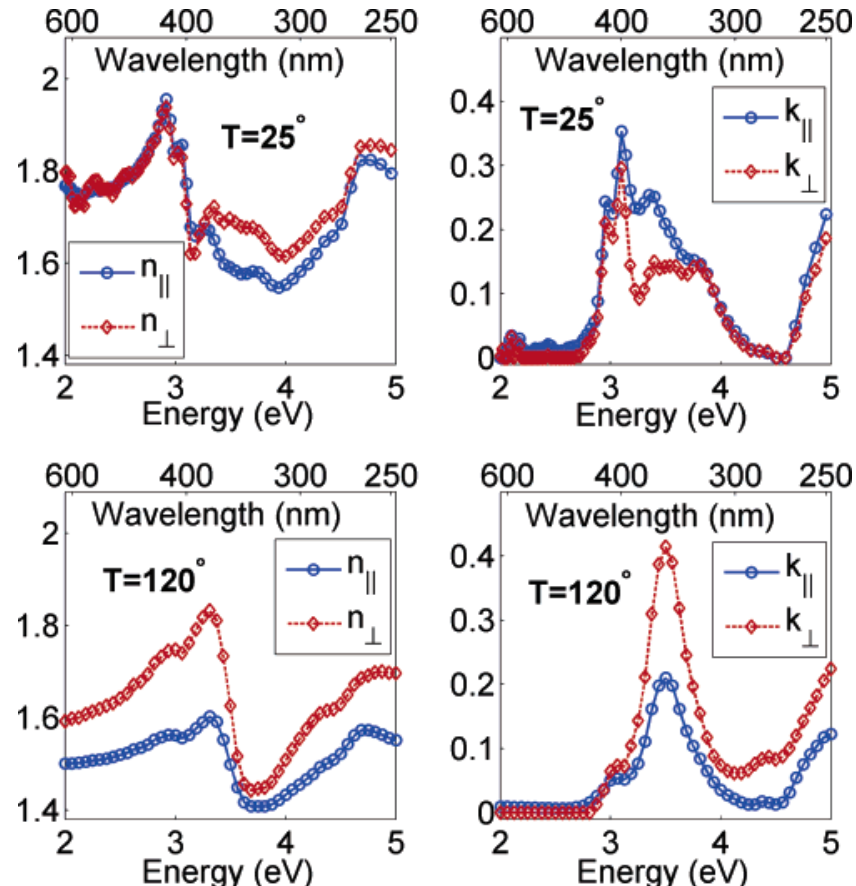

Figure 6. Spectral dependence of the complex refractive index $N=$ $n+i k$ parallel and perpendicular to the cast direction in the high- and low-temperature phases of the film. The appearance of a strongly anisotropic absorption peak at $3.5 \mathrm{eV}$ in the $T=120{ }^{\circ} \mathrm{C}$ phase is attributed to the reorientation of the discotic molecules.

constants depends on how accurately the film thickness was determined and the validity of neglecting roughness effects in the model. Numerical fitting for the optical constants using a film thickness $1 \mathrm{~nm}$ different from the one found above gives qualitatively the same spectra, only shifted by a few percent in absolute value. Accurate optical constants were difficult to extract because of the films being very thin and strong parameter correlations in the model.

An important observation is that the high-temperature phase has a high birefringence $\left|n_{\|}-n_{\perp}\right|$ of about 0.10 to 0.15 in the visual region of the spectrum $(<3.1 \mathrm{eV})$, which simultaneously has a low absorption. The most prominent change in the spectra going from $T=25^{\circ} \mathrm{C}$ to $T=120{ }^{\circ} \mathrm{C}$ is the appearance of a strong $\pi-\pi^{*}$ absorption peak at $3.5 \mathrm{eV}$ in the high-temperature phase, which is characteristic of the aromatic rings of HBC. The absorption is anisotropic with a dichroic ratio of about 2, as estimated from the ratio of $k_{\|}$and $k_{\perp}$. As expected, the curves are smoother in the mesophase where the contributions from vibronic states are absent.

\section{Discussion}

A Crystalline-Crystalline Transition $\left(T \approx 42{ }^{\circ} \mathrm{C}\right)$. The structure investigations show that the molecules are highly ordered in the as-cast phase, as evidenced also by the many higher-order reflections in Figure $2 \mathrm{a}$ being characteristic for a pseudo single-crystal structure. In a previous article on the ascast structure of $\mathrm{HBC}-\mathrm{C}_{12} \mathrm{H}_{25}$, we describe the dense and rather crystalline packing of the alkyl side chains, being extended parallel to the substrate. ${ }^{16}$ Evidently, the zone casting technique promotes this low-energy structure to be formed rather than the disordered state usually associated with alkyl chains. It has been reported previously that differential scanning calorimetry (DSC) shows a weak endothermic peak at $\sim 42{ }^{\circ} \mathrm{C} .{ }^{15}$ From the diffraction data, it is clear that a phase transition takes place at about this temperature, cf., Figures 2 and 3. Most importantly, the ED image in Figure $2 \mathrm{~b}$ still shows a distinct $h 01$ line, suggesting that this is a $\mathrm{Cr}-\mathrm{Cr}$ phase transition. According to Figure 5, this phase transition does not appear to significantly affect the optical properties of the film. Keeping in mind the surprising observation of the well-ordered side chains and the fact that alkyl chains are optically inactive, it seems reasonable to conclude that this phase transition mainly involves reorganization and increased mobility of the alkyl chains while only slightly perturbing the organization of the aromatic disks.

The $\mathbf{C r}-\mathbf{L C}$ Transition $\left(\boldsymbol{T} \approx 90{ }^{\circ} \mathbf{C}\right)$. It is of particular interest to watch the evolution of the $h 01$ line in Figure 2. The very existence of these mixed index reflections implies a proper crystalline structure, having registry between disks of neighbor- 
a)

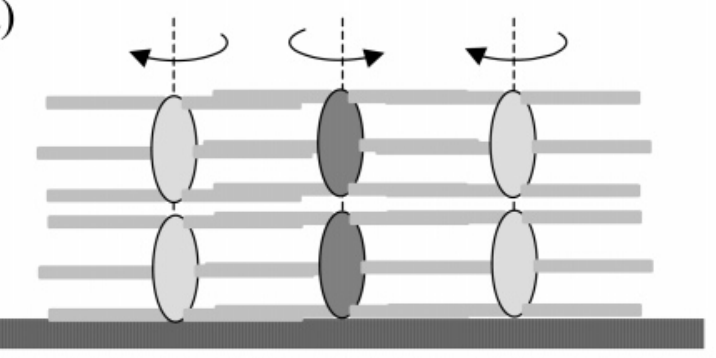

b)

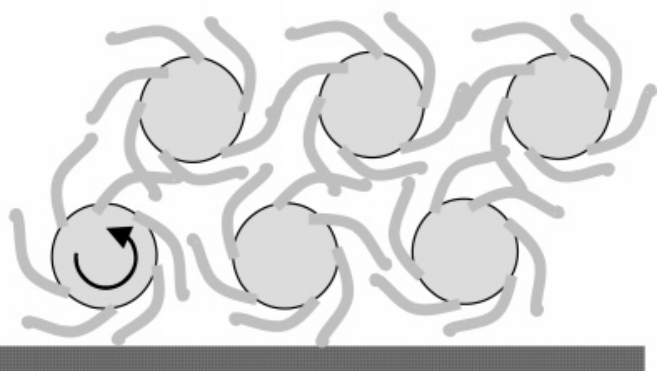

Figure 7. Schematic showing the $\mathrm{HBC}-\mathrm{C}_{12} \mathrm{H}_{25}$ structure viewed along the columns. (a) The as-cast structure with extended highly ordered side chains. Because of the $\sim 40^{\circ}$ rotation indicated by the arrows, the disks appear as ellipses. (b) The liquid crystalline state, with disordered chains in a $\mathrm{Col}_{\text {hex }}$ arrangement. From NMR, it is known that the molecules have a tendency to rotate as suggested by the arrow.

ing columns. The molecular form factor ${ }^{21}$ of the discotics acts like a "torch" illuminating regions in reciprocal space in the direction of the disk normal. The intensity distribution of the $h 01$ reflections is important for determining the $\sim 40^{\circ}$ rotation angle of the discotics within the columns. ${ }^{16}$ In the LC phase (Figure 2c,d), there are no mixed index crystalline reflections, and spatially slow variations in the electron density distribution are implied by the few higher-order reflections. However, the characteristic molecular spacing and the form factor result in two weak and broad off-meridional scattering features, corresponding to periodicities of $3.7 \AA$. The position of the peaks suggests that the disks are tilted by about $30^{\circ}$. Intriguingly, it appears that with higher temperature the disks rotate gradually toward a fully cofacial arrangement, with a stacking distance of $3.5 \AA$.

One might anticipate that the structure changes first from being orthorhombic (crystalline) to a rectangular columnar LC phase with tilted molecules, and at a higher temperature form a $\mathrm{Col}_{\text {hex }} \mathrm{LC}$ phase with cofacial molecules. However, from the observations presented here, we have a different interpretation: At the phase transition, the columns already existing in the crystalline phase slide across each other to form a $\mathrm{Col}_{\text {hex }} \mathrm{LC}$ phase with tilted molecules. At yet higher temperatures, the tilt is continuously reduced to form a cofacial $\mathrm{Col}_{\text {hex }}$ phase. Both the herringbone structure with ordered side chains and the LC $\mathrm{Col}_{\text {hex }}$ arrangement are shown schematically in Figure 7. The strong increase of optical anisotropy observed at the $\mathrm{Cr}-\mathrm{LC}$ phase transition can be explained by the intracolumnar reorganization of the aromatic $\mathrm{HBC}$ core molecules. In the herringbone phase, the disks of adjacent columns, and thus their dipole moments, are oriented at approximately $90^{\circ}$ to each other, effectively giving an optically isotropic phase. Conversely, for the hexagonal liquid-crystal phase, all dipole moments align in essentially the same direction, thereby reinforcing each other and giving a higher degree of optical anisotropy. ${ }^{16-18}$ The gradual increase of anisotropy with higher temperature above the phase transition is consistent with the mentioned tendency of an increasingly cofacial molecular arrangement with the aromatic ring normals orienting along the cast direction. The structural changes suggested in Figure 7 lead to a substantial increase of film thickness, or equivalently, a reduction in density. According to the $\mathrm{X}$-ray diffraction measurements, the spacing along b increases from $19.6 \AA$ (as cast films) to $27.8 \AA \times$ sin $60^{\circ} \approx 24.1 \AA$. The film thickness as measured by ellipsometry was $19.5 \mathrm{~nm}$ for the as-cast film. Coincidentally, this corresponds almost exactly to ten molecular layers. By the size of the unit cell, these ten layers have a predicted total thickness of $24.1 \mathrm{~nm}$ in the LC phase. In comparison with the $23.5 \mathrm{~nm}$ actually measured, this is another example of the good correspondence between our diffraction and optical measurements.

A plausible explanation for the expansion is the increased mobility of the molecules. From NMR studies, it has been concluded that the molecules rotate about their sixfold symmetry axis in the liquid crystalline phase. ${ }^{15}$ It is instructive to consider that this rotation will give the molecules an effectively circular disk appearance. The interpretation of circular disks is further strengthened by the $\chi$-scans shown in Figure 4 . The apparently perfect hexagonal packing is conceptually associated with circular shapes, as illustrated schematically in Figure $7 \mathrm{~b}$. Because these films are very thin and rather rough, it is not clear to the authors whether the increase in thickness is accompanied by lateral contractions or increased roughness.

As seen from the experimental data, there is a large degree of hysteresis. The alignment acquired during the zone-casting processing is unique. Heating of the sample has the effect of eliminating the structure history. Maybe surprisingly, the preferred orientation imposed by the zone-casting direction persists, cf., Figure 2, possibly because of the molecules being highly disc-shaped, thus making deviations from the columnar stacking energetically expensive. The tendency of arranging the $\mathrm{HBC}$ cores in a herringbone structure is retrieved upon re-entry to the crystalline phase, as judged by the low birefringence and the ED patterns resembling those of the as-cast phase. It appears statistically unlikely to return to the detailed zone-cast structure, and the measurements show only a partial recovery of the initial structure.

\section{Conclusions}

Using electron diffraction, grazing incidence X-ray diffraction, and variable angle spectroscopic ellipsometry, we have made a thorough investigation of the temperature-induced structural changes in thin films of zone-cast $\mathrm{HBC}-\mathrm{C}_{12} \mathrm{H}_{25}$. A $\mathrm{Cr}-\mathrm{Cr}$ transition is found at about $42{ }^{\circ} \mathrm{C}$, and a $\mathrm{Cr}-\mathrm{LC}$ transition is found at a higher temperature of around $90{ }^{\circ} \mathrm{C}$. Our studies provide new insights into the structure of the $\mathrm{LC}$ phase, which is found to be columnar hexagonal. The $\mathrm{Cr}-\mathrm{LC}$ transition is associated with a change of the disk rotation angle within the columns, from $\sim 40^{\circ}$ to $\sim 30^{\circ}$, which gives a strong increase of the optical anisotropy. At yet higher temperatures in the mesophase, evidence is found for a gradual reduction of this angle toward cofacially oriented disks. Also in the hightemperature state, a high degree of biaxial preferred orientation of the molecules is discovered. The $\mathrm{Cr}-\mathrm{LC}$ transition has a large hysteresis, and the extraordinarily high order of the ascast films is not fully retrieved after cooling back to room temperature. The anisotropic optical dispersion of these materials was mapped with unprecedented accuracy. We believe that these new insights will prove important to the continued progress of discotics for advanced applications.

Acknowledgment. We thank Dr. Bo Wegge Laursen for useful discussions and the HASYLAB staff for technical 
assistance. DanSync and the Danish Technical Research Council (STVF) are gratefully acknowledged for financial support.

\section{References and Notes}

(1) Malliaras, G.; Friend, R. Phys. Today 2005, 58, 53-58.

(2) Wu, J. S.; Watson, M. D.; Zhang, L.; Wang, Z. H.; Müllen, K. J. Am. Chem. Soc. 2004, 126, 177-186.

(3) Tchebotareva, N.; Yin, X. M.; Watson, M. D.; Samori, P.; Rabe, J. P.; Müllen, K. J. Am. Chem. Soc. 2003, 125, 9734-9739.

(4) Gearba, R. I.; Lehmann, M.; Levin, J.; Ivanov, D. A.; Koch, M. H. J.; Barbera, J.; Debije, M. G.; Piris, J.; Geerts, Y. H. Adv. Mater. 2003, 15, $1614-1618$

(5) Bunk, O.; Nielsen, M. M.; Sølling, T. I.; van de Craats, A. M.; Stutzmann, N. J. Am. Chem. Soc. 2003, 125, 2252-2258.

(6) Pisula, W.; Kastler, M.; Wasserfallen, D.; Pakula, T.; Müllen, K. J. Am. Chem. Soc. 2004, 126, 8074-8075.

(7) Liu, C. Y.; Bard, A. J. Chem. Mater. 2000, 12, 2353-2362.

(8) Laursen, B. W.; Norgaard, K.; Reitzel, N.; Simonsen, J. B.; Nielsen, C. B.; Als-Nielsen, J.; Bjornholm, T.; Sølling, T. I.; Nielsen, M. M.; Bunk, O.; Kjaer, K.; Tchebotareva, N.; Watson, M. D.; Mullen, K.; Piris, J. Langmuir 2004, 20, 4139-4146.

(9) Breiby, D. W.; Samuelsen, E. J.; Konovalov, O.; Struth, B. Langmuir 2004, 20, 4116-4123.

(10) Samuelsen, E. J.; Aasmundtveit, K. E.; Breiby, D. W. In Electronic and Optical Properties of Conjugated Molecular Systems in Condensed Phases; Hotta, S., Ed.; Research Signpost: Kerala, India, 2003.

(11) Pisula, W.; Tomovic, Z.; Stepputat, M.; Kolb, U.; Pakula, T.; Müllen, K. Chem. Mater. 2005, 17, 2641-2647.
(12) Pisula, W.; Menon, A.; Stepputat, M.; Lieberwirth, I.; Kolb, U.; Tracz, A.; Sirringhaus, H.; Pakula, T.; Müllen, K. Adv. Mater. 2005, 17 , 684-689.

(13) Debije, M. G.; Piris, J.; de Haas, M. P.; Warman, J. M.; Tomovic, Z.; Simpson, C. D.; Watson, M. D.; Müllen, K. J. Am. Chem. Soc. 2004, $126,4641-4645$

(14) Fechtenkötter, A.; Saalwachter, K.; Harbison, M. A.; Müllen, K.; Spiess, H. W. Angew. Chem., Int. Ed. 1999, 38, 3039-3042.

(15) Fischbach, I.; Pakula, T.; Minkin, P.; Fechtenkötter, A.; Müllen, K.; Spiess, H. W.; Saalwachter, K. J. Phys. Chem. B 2002, 106, 64086418.

(16) Breiby, D. W.; Bunk, O.; Pisula, W.; Sølling, T. I.; Tracz, A.; Pakula, T.; Müllen, K.; Nielsen, M. M. J. Am. Chem. Soc. 2005, 127 , $11288-11293$.

(17) Piris, J.; Debije, M. G.; Stutzmann, N.; Laursen, B. W.; Pisula, W.; Watson, M. D.; Bjørnholm, T.; Müllen, K.; Warman, J. M. Adv. Funct. Mater. 2004, 14, 1053-1061.

(18) Piris, J.; Pisula, W.; Tracz, A.; Pakula, T.; Müllen, K.; Warman, J. Liq. Cryst. 2004, 31, 993-996.

(19) Stabel, A.; Herwig, P.; Müllen, K.; Rabe, J. P. Angew. Chem., Int. Ed. Engl. 1995, 34, 1609-1611.

(20) Tracz, A.; Jeszka, J. K.; Watson, M. D.; Pisula, W.; Müllen, K.; Pakula, T. J. Am. Chem. Soc. 2003, 125, 1282-1683.

(21) Als-Nielsen, J.; McMorrow, D. Modern X-ray Physics; John Wiley \& Sons, Ltd.: West Sussex, 2004.

(22) Azzam, R. M. A.; Bashara, N. M. Ellipsometry and Polarized Light, 4th ed.; Elsevier: New York, 1999. 